\title{
A fast-running CFD formulation for unsteady ship maneuvering performance prediction
}

\author{
M.O. Woolliscroft*, K.J. Maki \\ Department of Naval Architecture and Marine Engineering, University of Michigan, Ann \\ Arbor MI, 48109, USA
}

\begin{abstract}
Maneuvering prediction tools are important resources for naval and commercial ship designers. They can enable designers to determine maneuvering characteristics of a design prior to construction or be useful in redesign if a ship is underperforming. Numerical methods allow for multiple hulls to be compared digitally, so a certain level of optimization can be achieved through evaluating trade-offs among designs before construction. In this paper, a novel maneuvering prediction method is presented that seeks to provide the accuracy available from todays state-of-the-art numerical viscous-flow tools but with significantly reduced computational cost. The numerical formulation is based on the unsteady Reynolds-averaged Navier-Stokes (URANS) equations, and the unsteady ship generated waves are represented by the linear free-surface boundary conditions. The URANS equations are solved on an unstructured finite-volume discretization of the fluid domain around the hull and its appendages. The linearized free-surface approximation accounts for first-order wave effects while reducing the necessary extent of the computational domain and level of grid refinement required by fully nonlinear methods that employ an interface-capturing technique. The resulting formulation provides a marked improvement in computational efficiency with respect to nonlinear methods and can empower naval architects to obtain accurate results earlier in the design process.
\end{abstract}

Keywords: maneuvering prediction, early-stage design, linearized free-surface, URANS

\footnotetext{
${ }^{*}$ Corresponding author

Email address: mowoolli@umich.edu (M.O. Woolliscroft)

Preprint submitted to Ocean Engineering
}

February 12, 2016 


\section{Introduction}

Maneuvering performance is an important facet of ship design. A large number of performance quantities are considered throughout the design process, and many of them are interdependent such that changes in one area propagate and cause expensive and time consuming re-evaluation of the other performance metrics. This is clearly seen in the classical iterative design spiral, where areas such as hull form, powering, resistance, seakeeping, arrangements, and cost are analyzed multiple times as a design becomes refined and converges to a detailed point design.

Maneuverability is an example of a performance metric that is connected to the many other areas. While the design spiral is the classical way to consider design creation, a design network is a newer alternative that gives additional insight to the complex interactions of different performance attributes (Gillespie et al., 2013). This new perspective places maneuvering as a node within the design network, where the dependencies range from ship arrangements, resistance, prime-mover selection, economics, etc.

Maneuvering attributes have far-reaching consequences throughout the design, but even within the specific maneuvering evaluation there are many interdependent factors. The hydrodynamic interaction between the hull, propellers, rudders, and other control surfaces are fully coupled and challenging to predict because of issues such as the high-Reynolds number operation of ships (wide range of length scales) and the delicate interaction between ship waves and the turbulent flow that can separate along the hull and strongly influence the propeller and rudder performance.

The goal of a naval architect is to evaluate the trade offs within the design space and deliver an optimal solution. Nevertheless, real-world constraints such as schedules and budgets often lead to an incomplete exploration of novel hull forms and instead, historical hull forms are often used. The International Maritime Organization mandates that new designs pass several maneuvering criteria. In general, this includes the ability to stop or turn within a certain distance, and the like. Therefore, maneuvering assessment is viewed as a necessary step in the design process. At times, an existing hull may fully satisfy the requirements of a new design, but this approach lacks a motivated effort to evolve the designs of the largest transportation vehicles in the world. The lack of accurate and efficient design tools hinders the evolution of a ship 
design towards an optimal form. On rare occasions, a more original design may be evaluated with high-fidelity methods such as computational fluid dynamics (CFD) or a new set of physical experiments, but the determination of the maneuvering characteristics with these methods is a difficult task itself. This fact sets maneuvering prediction apart from the other aspects of ship design; although it is a crucial node in the design network, it is unique because it is less frequently analyzed prior to the detailed design stage due to the resources required for high-fidelity maneuvering predictions.

Currently, maneuvering prediction capabilities consist of geometrically scaled model tests and a variety of numerical methods. Model tests provide valuable predictive information about the performance of a design, but they require expensive facilities, models, and instruments, and a procedure to account for scaling effects (Cope, 2012; Ueno et al., 2014). Numerical methods vary from inviscid potential flow to multiphase CFD codes. Historically, the velocity potential framework provides solutions to ship-motions problems, ranging from stability assessment to the overtaking of one ship by another (Sclavounos and Thomas, 2007; Newman and Tuck, 1974). Recent examples of real-time maneuvering simulation based on potential flow modeling can found in (Lindberg et al., 2013) (Pinkster and Bhawsinka, 2013) (Zhou et al., 2014). However, potential flow codes inherently lack the ability to capture viscous separation which is especially significant near the hull appendages that influence maneuverability. CFD and model tests can be used to obtain viscous predictions on hulls with appendages (Broglia et al., 2013) (Carrica et al., 2012), but such methods of obtaining corrections are costly and time-consuming. Most designers do not have the computational resources necessary for CFD simulations of time-dependent maneuvers.

Another powerful approach to maneuvering simulation is the systemsbased methods. Equation-of-motion coefficients are obtained from physical experiments or CFD to predict maneuvering capabilities (Toxopeus, 2009). The main advantage of these methods is that after the coefficients are determined, the maneuvering equations can be solved in a very efficient manner.

The main drawbacks of existing maneuvering prediction techniques make them limited for use in early-stage conceptual design. They are used in the final stages, where changes to the ship are so expensive that they are avoided if it all possible. As a result, while many other aspects of a design are improved at each iteration, optimization of the maneuvering characteristics is rarely pursued. Therefore, it is an important endeavor to provide efficient and accurate tools that allow for a broad exploration of the design space and 
the ability to develop optimized designs within realistic time and monetary constraints.

\section{Computational method}

\subsection{Governing equations}

The linearized free-surface conditions for maneuvering in deep and calm water have been studied comprehensively for a potential-flow framework, but much less so for viscous flows. In this section, the governing equations are presented, and the linearized free-surface conditions are derived for a viscous and turbulent flow starting from the nonlinear free-surface conditions.

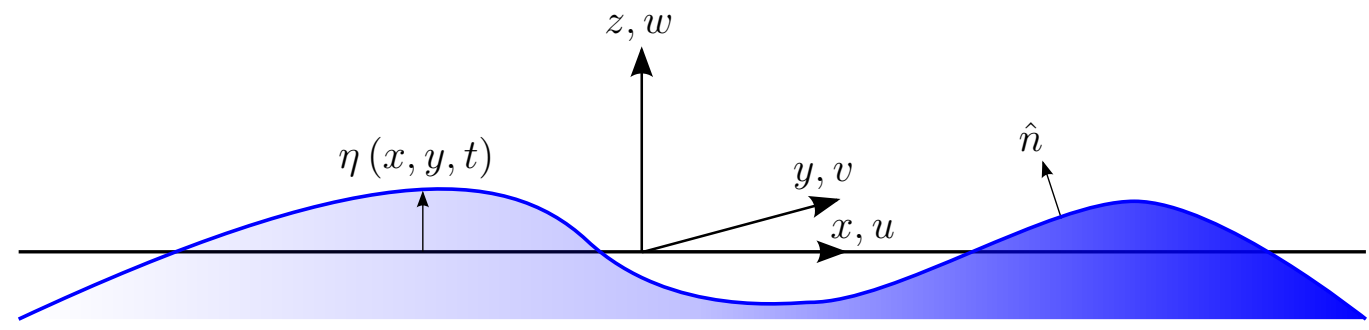

Figure 1: Nonlinear free-surface example

The governing equations and boundary conditions are presented in two reference frames. The first is an Earth-fixed reference frame (Fig. 1), where $x, y$, and $z$ are the three Cartesian coordinates and the origin is fixed to the Earth in the calm-water plane. The fluid velocity vector, $\vec{u}=u \hat{i}+v \hat{j}+w \hat{k}$, contains components which act along the three spatial axes. The free-surface elevation $\eta$ is a function of $x, y$, and time, $t$. The unit vector $\hat{n}$ is normal to the free-surface and points out of the water. The second reference frame is fixed to the ship, and is denoted with a prime as $x^{\prime}, y^{\prime}$, and $z^{\prime}$. In the ship-fixed frame the fluid velocity is $\vec{u}^{\prime}=u^{\prime} \hat{i}+v^{\prime} \hat{j}+w^{\prime} \hat{k}$. The ship does not move vertically, so the ship velocity vector is $\vec{U}_{\text {ship }}=U_{\text {ship }} \hat{i}+V_{\text {ship }} \hat{j}$.

The equations governing conservation of momentum are the incompressible URANS equations,

$$
\begin{gathered}
\frac{d}{d t} \int_{V} \rho \vec{u} d V+\int_{S} \rho \vec{u} \vec{u} \cdot \hat{n} d S=-\int_{V} \nabla \bar{p} d V+\int_{S} \mu_{\mathrm{eff}}\left(\nabla \vec{u}+\nabla \vec{u}^{T}\right) \cdot \hat{n} d S \\
\int_{S} \vec{u} \cdot \hat{n} d S=0
\end{gathered}
$$


where the effective viscosity is the sum of the molecular and turbulent viscosities $\mu_{\text {eff }}=\mu+\mu_{t}$. Control volumes and control surfaces are denoted with $V$ and $S$, respectively.

The kinematic condition states that the free-surface is a material surface, the location of which can be defined with a function:

$$
F(x, y, z, t)=z-\eta(x, y, t)=0
$$

The value of Eq. 3 is always zero on the surface. Hence, the total derivative of the function is also zero on the surface:

$$
\frac{D F}{D t}=\frac{\partial F}{\partial t}+\vec{u} \cdot \nabla F=0
$$

This condition is first expressed in the frame moving with the ship, as shipgenerated waves are steady from this perspective for simple problems such as forward-speed resistance. This can be done by using the relations between the ship-frame and Earth-frame and evaluating the total derivative of the freesurface function (Eq. 4) in terms of $\eta$. The position and velocity relations between the two frames are:

$$
\begin{aligned}
\vec{x} & =\vec{x}^{\prime}+\int_{0}^{t} \vec{U}_{\text {ship }}(t) d t \\
\vec{u} & =\vec{u}^{\prime}+\vec{U}_{\text {ship }}
\end{aligned}
$$

The total derivative of the free-surface function with respect to the moving ship-frame is expressed in Eq. 7:

$$
\frac{D F}{D t}=\eta_{t}+\left(-U_{\text {ship }}+u\right) \eta_{x^{\prime}}+\left(-V_{\text {ship }}+v\right) \eta_{y^{\prime}} \quad=w \quad \text { Ship-fixed }
$$

The stationary, Earth-fixed representation of the total derivative of the freesurface function is obtained by having the ship velocity go to zero, $\vec{U}_{\text {ship }}=\overrightarrow{0}$ :

$$
\frac{D F}{D t}=\eta_{t}+u \eta_{x}+v \eta_{y} \quad=w \quad \text { Earth-fixed }
$$

Eqs. 7 and 8 are the unsteady nonlinear kinematic boundary conditions with respect to the two frames. The linearization of each condition stems from 
small value assumptions. For maneuvering flows, it is assumed that $\vec{u}$ is small, and the ship-generated waves have small slopes and small heights. The linearized kinematic conditions are:

$$
\begin{array}{lr}
w=\eta_{t}-U_{\text {ship }} \eta_{x^{\prime}}-V_{\text {ship }} \eta_{y^{\prime}} & \text { Ship-fixed } \\
w=\eta_{t} & \text { Earth-fixed }
\end{array}
$$

Eqs. 9 and 10 are satisfied on the static $z=0$ calm-water plane. This simplifying assumption gives rise to computational savings by avoiding the need for mesh deformation required by nonlinear surface tracking methods. Lastly, for cases in which the flow is steady in the ship-fixed coordinate, the steady kinematic condition can be used as:

$$
w=-U_{\text {ship }} \eta_{x^{\prime}}-V_{\text {ship }} \eta_{y^{\prime}} \quad \text { Steady ship-fixed }
$$

The dynamic free-surface boundary condition is derived by invoking a zero total stress condition on the free-surface. It is assumed that surface tension is insignificant for ship waves from the maneuvering point-of-view such that it can be neglected, and the total stress tensor is written as,

$$
\overline{\bar{\sigma}}=-(p-\rho g \eta) \overline{\bar{I}}+\overline{\bar{\tau}}
$$

where $p$ is the hydrodynamic pressure, $\overline{\bar{I}}$ is the identity tensor, and $-\rho g \eta$ is the hydrostatic pressure. The incompressible viscous stress tensor is expressed as:

$$
\overline{\bar{\tau}}=\mu\left(\nabla \vec{u}+(\nabla \vec{u})^{T}\right)
$$

The superscript $T$ indicates the tensor transpose operation. The zero total stress boundary condition implies $\overline{\bar{\sigma}} \cdot \hat{n}=\overrightarrow{0}$. This condition can be expressed in several ways. First, the zero-stress condition is written explicitly in terms of the free-surface elevation $\eta$. One can non-dimensionalize the dynamic conditions using the ship length and forward speed as the length and velocity scales to characterize the problem. Collecting appropriate terms for the Froude and Reynolds numbers (based on the ship length) results in the following description: 


$$
\begin{aligned}
& \left(\tilde{p}-F r^{-2} \tilde{\eta}\right) \tilde{\eta}_{\tilde{x}}-2 R e^{-1} \tilde{u}_{x} \tilde{\eta}_{\tilde{x}}-R^{-1}\left(\tilde{u}_{y}+\tilde{v}_{x}\right) \tilde{\eta}_{\tilde{y}}+\operatorname{Re}^{-1}\left(\tilde{u}_{z}+\tilde{w}_{x}\right)=0 \\
& \left(\tilde{p}-F r^{-2} \tilde{\eta}\right) \tilde{\eta}_{\tilde{y}}-\operatorname{Re}^{-1}\left(\tilde{v}_{x}+\tilde{u}_{y}\right) \tilde{\eta}_{\tilde{x}}-2 R e^{-1} \tilde{v}_{y} \tilde{\eta}_{\tilde{y}}+R e^{-1}\left(\tilde{v}_{z}+\tilde{w}_{y}\right)=0 \\
& -\left(\tilde{p}-F r^{-2} \tilde{\eta}\right)-\operatorname{Re}^{-1}\left(\tilde{w}_{x}+\tilde{u}_{z}\right) \tilde{\eta}_{\tilde{x}}-\operatorname{Re}^{-1}\left(\tilde{w}_{y}+\tilde{v}_{z}\right) \tilde{\eta}_{\tilde{y}}+2 R e^{-1} \tilde{w}_{z}=0
\end{aligned}
$$

This dimensionless form allows for a quantitative examination of the importance of each term in the zero total stress condition. In addition to being nonlinear, these equations are coupled by the variables $u, v, w, p$, and $\eta$. It is assumed that the ship-generated waves caused by maneuvering predominantly have small slopes and small heights, where $\eta_{x}, \eta_{y} \ll 1$ and $\eta \ll L_{\text {ship }}$. Additionally, even at model scale, ships operate at Reynolds numbers exceeding $\mathcal{O}\left(10^{5}\right)$ whereas the Froude number is often $\mathcal{O}\left(10^{-1}\right)$. Therefore, each viscous term in the dynamic conditions is neglected and the resulting condition is a single, linearized, zero total pressure equation (shown again in dimensional form):

$$
p-\rho g \eta=0
$$

Eq. 15 is used as the pressure boundary condition with the linearized URANS method and is satisfied on the $z=0$ plane.

The body boundary condition for the fully nonlinear problem states that the velocity of the fluid is equal to the velocity of the body:

$$
\vec{u}=\vec{U}_{\text {body }}
$$

One of the important benefits of following a linearized approach is the reduction of computational cells that are required to resolve the free-surface solution. In the current implementation, the computational domain does not extend beyond the $z=0$ calm-water plane. As such, the ship and grid move together in the horizontal plane, and the body boundary condition can be satisfied exactly for the surge, sway, and yaw degrees of freedom.

\subsubsection{ALE Form of Equations}

The governing equations have been presented, and are solved, in an inertial, Earth-fixed reference frame. The moving grid necessitates an arbitrary 
Lagrangian-Eulerian (ALE) description of the governing equations for numerical solution. Equations are solved for each computational cell that has volume $V$ and is bounded by the surface $S$ with outward normal $\hat{n}$. $S_{o}$ is the portion of the boundary of a computational cell that is adjacent to the plane $z=0$, and $l$ is the contour of this area. The development of the ALE form of the kinematic free-surface boundary condition begins with the Leibniz integral rule applied over a surface to the time-derivative of $\eta$ on the right-hand side of Eq. 10,

$$
\frac{\partial}{\partial t} \int_{S_{o}(t)} \eta d S=\int_{S_{o}(t)} \frac{\partial \eta}{\partial t} d S+\int_{l(t)} \eta \frac{\partial \vec{x}_{\mathrm{mesh}}(t)}{\partial t} \cdot \hat{n} d l
$$

where,

$$
\frac{\partial \vec{x}_{\text {mesh }}(t)}{\partial t}=\vec{U}_{\text {mesh }}=U_{\text {mesh }} \hat{i}+V_{\text {mesh }} \hat{j}+0 \hat{k}
$$

The mesh motion gives rise to a convective term in the ALE formulation of the kinematic free-surface boundary condition. This is consistent with the convective term in the ship-fixed condition in Eq. 9 because $\vec{U}_{\text {mesh }}$ takes the place of $\vec{U}_{\text {ship }}$. The form of the condition that is suitable for numerical solution on a moving grid is:

$$
\frac{\partial}{\partial t} \int_{S_{o}(t)} \eta d S-\int_{l(t)} \eta \vec{U}_{\mathrm{mesh}} \cdot \hat{n} d l=\int_{S_{o}(t)} w d S
$$

Similarly, the ALE form of the momentum and continuity equations is,

$$
\begin{gathered}
\frac{d}{d t} \int_{V} \rho \vec{u} d V+\int_{S} \rho \vec{u} \vec{u}_{\mathrm{rel}} \cdot \hat{n} d S=-\int_{V} \nabla \bar{p} d V+\int_{S} \mu_{\mathrm{eff}}\left(\nabla \vec{u}+\nabla \vec{u}^{T}\right) \cdot \hat{n} d S \\
\int_{S} \vec{u}_{\mathrm{rel}} \cdot \hat{n} d S=0
\end{gathered}
$$

where,

$$
\vec{u}_{\text {rel }}=\vec{u}-\vec{U}_{\text {mesh }}
$$




\subsection{Numerical aspects}

The linearized URANS formulation is solved using a custom finite volume CFD algorithm based within the OpenFOAM $\mathrm{C}++$ library. It consists of solutions to the URANS equations and linear free-surface conditions. All flow variables are collocated at cell centers of the polyhedral finite-volume mesh. At the free-surface, values for the wave elevation, $\eta$, are solved at face centers of the polygonal boundary mesh.

Results discussed in this paper are obtained on unstructured grids. A PISO-like algorithm is used to solve for pressure and velocity. Time discretization is performed with a first-order Euler implicit scheme. A secondorder linear upwind scheme is used for convective terms. The SpalartAllmaras turbulence model is used with an adaptive wall-function based on the Spalding universal law-of-the-wall.

The entire computational domain is moved with rigid body motion in the horizontal plane. The ship motion is described in an inertial, Earth-fixed reference frame. This approach allows for a natural description of the acceleration of the body from rest and avoids issues related to an impulsive start which is discussed in Sec 3.1. Furthermore, it closely resembles the actual motions in a physical setting (which in this validation is a towing tank). While the entire grid undergoes rigid body motion, propellers and rudders can rotate relative to the body with a sliding mesh approach. A cylinder enclosing a propeller or rudder rotates independently from the remainder of the mesh.

Fig. 2 describes the steps of the algorithm. The starting point for the custom solver developed in this work is the pimpleDyMFoam solver, and the unique steps that add the linear unsteady free-surface capability are outlined in red. The solution of the momentum equations provides the vertical velocity which is used in the kinematic boundary condition. In a typical segregated manner, the pressure equation is then solved and used to correct the velocities at cell centers to achieve a discretely divergence-free velocity vector. Upon updating the boundary conditions over the computational domain, the pressure condition on the free-surface uses the wave elevation from the kinematic condition to apply the pressure boundary condition. Steady problems can be modeled by using time steps to dictate the number of iterations with no inner correctors, outer correctors, or time derivatives. For unsteady problems, time steps can dynamically adjust to a user-defined Courant restriction. Inner correctors can be used to solve the momentum equations multiple times within a single time step. Lastly, under-relaxation may be 
employed in combination with outer correctors for time-accurate solutions employing large time steps.

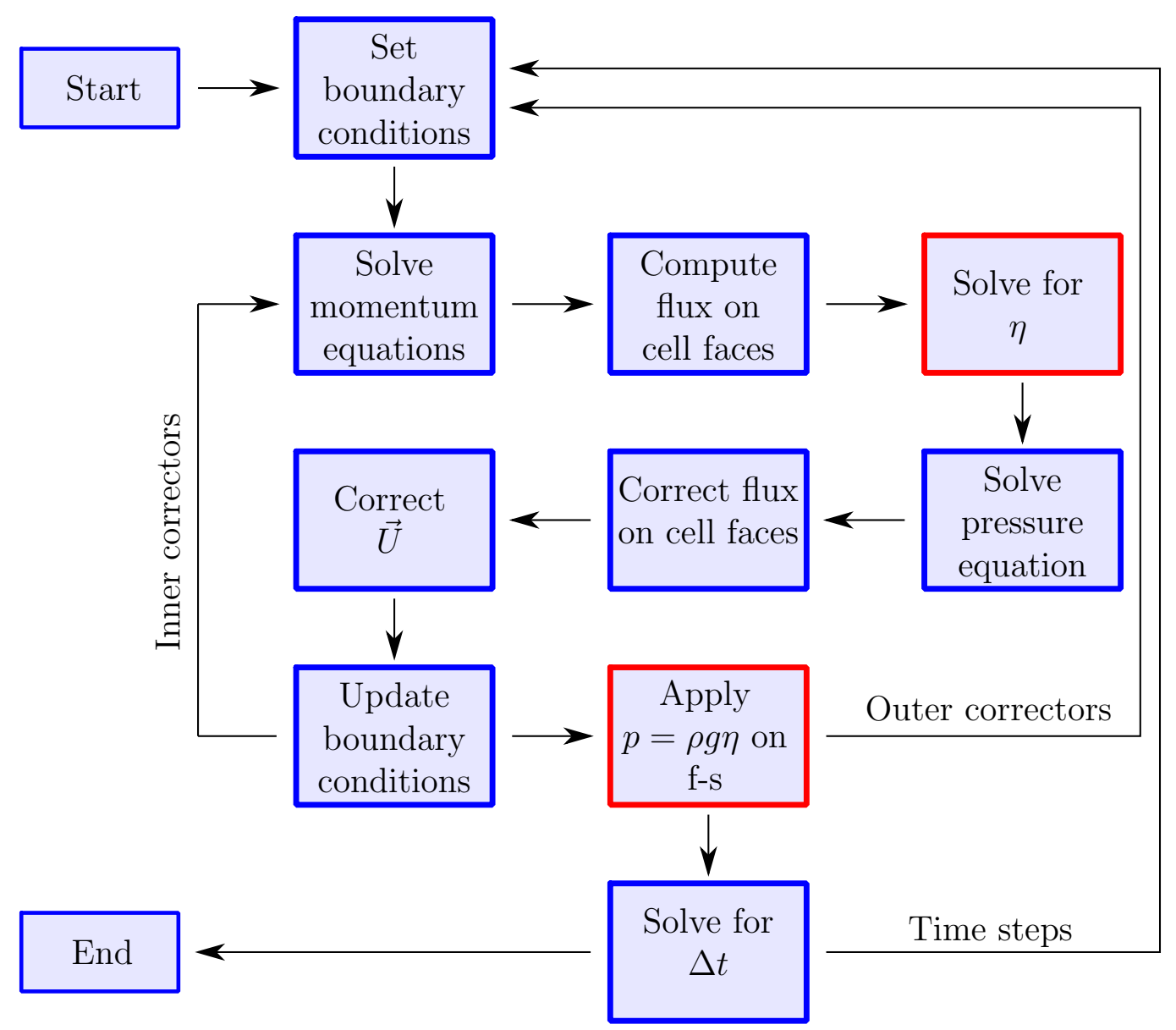

Figure 2: Steps of the linearized URANS algorithm

\section{Validation with Wigley Hull}

The governing equations previously described in this document are derived for the simulation of ship maneuvering and directly applicable to simulate planar motion mechanism (PMM) maneuvers. The equations are unsteady and written in an ALE fashion for an Earth-fixed description of the maneuvering problem. However, the general linearized free-surface approach is also well-suited for other hydrodynamics applications such as sub- 
merged hydrofoils, surface-piercing cylinders, submarines near or piercing the free-surface, submerged energy-capturing devices, and moonpool resonance (Woolliscroft and Maki, 2015).

Different applications may benefit from different reference frames to be selected for the solution algorithm. For instance, ship resistance and static drift problems can be solved in a ship-fixed reference frame with steady forms of the governing equations. Dynamic maneuvers such as pure sway and pure yaw benefit from an inertial, Earth-fixed reference frame with ALE descriptions of the governing equations. Three variations of the linearized URANS free-surface solver are tested in this section.

- The first numerical variation is a steady ship-fixed formulation, where time derivatives $\partial \vec{u} / \partial t$ and $\partial \eta / \partial t$ are assumed zero and absent from the discretized equations. The convective term in the kinematic condition (Eq. 11) arises from the coordinate-system shift instead of the ALE procedure.

- The second variation is unsteady and employs the ship-fixed frame of reference where $\partial \vec{u} / \partial t$ and $\partial \eta / \partial t$ are solved using a first-order Euler implicit scheme. This form of the solver is only used for cases when the body is not accelerating so that the source terms due to the acceleration of the coordinate system are zero. This requires an impulsive start.

- Lastly, an inertial Earth-fixed approach is used, where the ALE form of the governing equations is solved. Naturally, this approach is unsteady and requires inclusion of time-derivatives of flow variables. The ALE form of the Earth-fixed kinematic condition (Eq. 19) is solved in this variation.

The purpose of this study is to evaluate and compare the three variations of the linearized URANS problem for the purpose of ship maneuvering. The accuracy and computational expense is discussed for each variation.

\subsection{Resistance Tests}

Resistance tests of a Wigley hull with length $L=4$ meters are performed at several Froude numbers with three variations of the linearized free-surface tool. The hull has a length-to-beam ratio of $L / B=10$ and a beam-to-draft ratio of $B / D=1.6$. An equation for the hull form is shown 
in Eq. 23. The accuracy of the numerical predictions is determined by comparison with experimental measurements from the Ship Research Institute in Tokyo, Japan (Tanaka, 1983).

$$
y=\frac{B}{L}\left[1-\left(\frac{2 x}{L}\right)^{2}\right]\left[1-\left(\frac{z}{D}\right)^{2}\right]
$$

Although resistance is used to evaluate the method and its variations, consistency with maneuvering notation is maintained with the use of $X$ as the total resistance and $X^{\prime}$ as the dimensionless coefficient of total resistance. This and the lateral sway force, $Y^{\prime}$, and yaw moment, $N^{\prime}$, are calculated as:

$$
X^{\prime}, Y^{\prime}, N^{\prime}=\frac{X, Y, N / L}{0.5 \rho U_{\text {ship }}^{2} A_{o}}
$$

The ship length is $L$, and the lateral underwater area, $A_{o}=L D$ where $D$ is the draft.

A single structured grid consisting of approximately 120,000 cells is used for all simulations. The Spalart-Allmaras turbulence model is used with a universal wall-function. The lowest $y^{+}$values which correspond to $F r=0.1$ are $y_{\min }^{+}=21, y_{\text {mean }}^{+}=48$, and $y_{\max }^{+}=86$. A grid refinement study is performed in the section on maneuvering forces (Sec. 4).

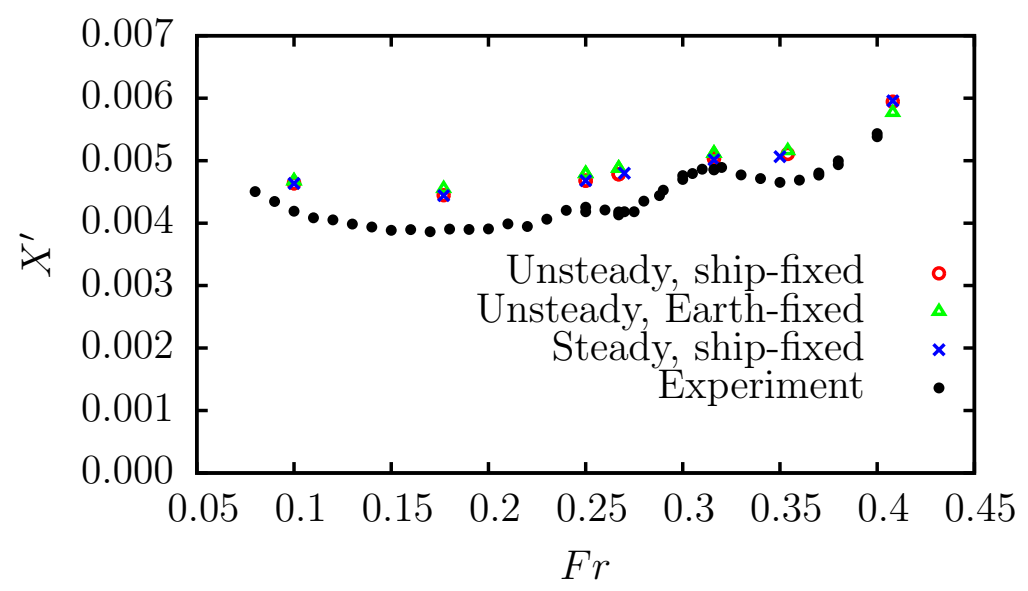

Figure 3: Resistance coefficients of experiments and three variations of the linearized freesurface method

The predicted resistance coefficients from each variation of the linearized free-surface solver and the physical experiments are shown in Fig. 3. All 
three variations of the linearized (U)RANS method predict similar values of the resistance coefficient at each respective Froude number. Although the numerical results differ by up to $15 \%$ compared to the experiments, the difference is always positive and reasonably consistent. There are several possible causes for the difference between the numerical predictions and the experimental measurements; grid refinement, turbulence modeling, and use of a wall-function can all play a role in the comparison error. The main focus here is to demonstrate the ability to consistently model steady, unsteady, non-inertial, and inertial problems in a RANS framework using a linearized free-surface.

The convergence of the resistance using the steady ship-fixed variation is presented in Fig. 4. 12,000 iterations are performed for each simulation. The resistance values corresponding to the highest and lowest Froude numbers need far fewer iterations to converge, but not all resistance values have converged for the midrange Froude numbers, especially $F r=0.32$ and $F r=0.35$. The oscillations associated with the midrange Froude numbers can be attributed to the impulsive start used in the simulations. The initial state from this impulsive start provides inexact values for the steady solution and leads to an oscillating wave. An iterative algorithm is used for the steady equations, but the iterative process acts as pseudo-time. Therefore, the error oscillates and decreases with additional iteration, akin to an unsteady algorithm converging over time.

The two variations of the unsteady description of the linearized URANS method - ship-fixed and Earth-fixed reference frames - are compared in Fig. 5. The difference that is highlighted here is the use of an impulsive start compared to a slow increase or ramp-up in velocity. The simulations corresponding to the ship-fixed reference frame use an impulsive start for the fluid velocity, where $U_{\text {ship }}=U_{\text {final }}$ for $t>0$. The Earth-fixed simulations use a half-cosine velocity ramp to describe speed of the ship (Eq. 25):

$$
U_{\text {ship }}= \begin{cases}\frac{1}{2} U_{\text {final }}\left(1-\cos \left(\frac{\pi t}{t_{\text {ramp }}}\right)\right), & \text { if } t \leq t_{\text {ramp }} \\ U_{\text {final }}, & \text { if } t>t_{\text {ramp }}\end{cases}
$$

The Earth-fixed simulations, which all employ a velocity ramp of 9 seconds, are shown to converge more quickly than the ship-fixed simulations that use an impulsive start. The impulsive start creates waves from reflection at the boundaries leading to oscillatory forces. The period of the oscillation is described by linear wave potential theory $T=8 \pi U / g$ (Wehausen and 
Laitone, 1960, pg 617), and the amplitude of the oscillation is proportional to the acceleration, being maximum for the impulsive start. The oscillations require long periods of time to dampen via the means of physical viscosity and numerical error. In fact, the ramp assists in speeding up convergence so well that an unsteady approach may require less CPU time to reach convergence than a steady approach. Normally, the steady resistance problem is an excellent candidate for the steady solver. The steady solution is appealing due computational effort (all time derivatives neglected) and a stationary mesh. On the other hand, the unsteady version may seem unnecessarily complex as it requires solutions to time derivatives, time-step size restrictions, and the cost of translating the computational mesh. It is possible to use relaxation techniques to improve the convergence of the steady solver, but the natural formulation of the unsteady problem allows for a Courant-number-based time-step-size restriction to control the convergence in an efficient manner. A summary comparing the computing time for this case appears in Table 1. 
Figure 5: Dashed lines - Unsteady ship-fixed without velocity ramp-up Solid lines - Unsteady Earth-fixed with velocity ramp-up

The general free-surface linearization in a RANS framework is shown to provide consistent results with a steady, unsteady ship-fixed, and unsteady Earth-fixed version. But, the overall goal at hand is for unsteady maneuvering simulations in the horizontal plane. Another advantage to an Earth-fixed frame of reference arises with additional motions of sway and yaw. With an Earth-fixed reference frame, a user may input the ship motions as they would be input on a towing tank carriage (forward speed, sway/yaw amplitude and frequency) while avoiding prescribing any time-dependent changes in the boundary conditions. For this reason, as well as those discussed previously, the maneuvering simulations presented in the following section are performed in an unsteady, Earth-fixed manner with a velocity ramp for the surge, sway, and yaw motions. 
Table 1: CPU time to convergence

\begin{tabular}{lccc}
\hline description & iterations/time & converged? & CPU hours \\
\hline steady & 12,000 iterations & no & 3.43 \\
unsteady & $15 \mathrm{~s}$ & yes & 2.66 \\
\hline
\end{tabular}

\section{Maneuvering Results}

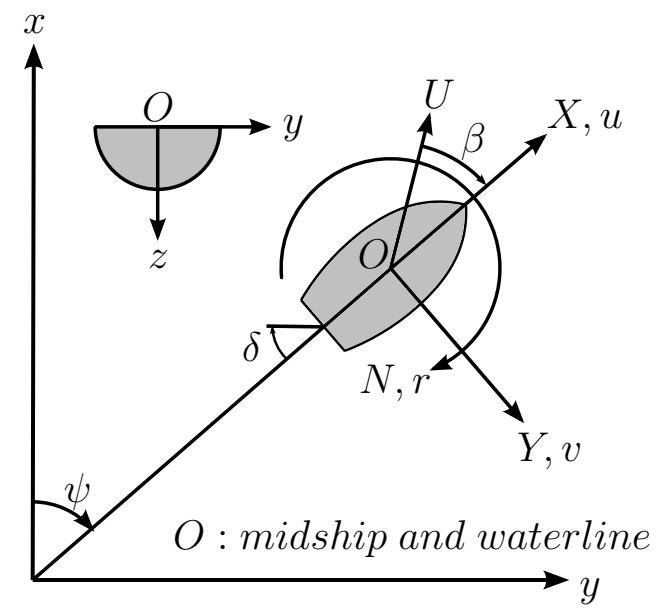

Figure 6: The maneuvering coordinate system

\subsection{Overview of test case}

PMM captive model tests are used to determine the accuracy of the linearized URANS method in the prediction of forces and moments. A version of the David Taylor Model Basin (DTMB) 5415 hull form is used. The model has length $L=3.048$ meters and is fitted with bilge keels but is otherwise unappended. The Froude number is 0.28 , and the length-based Reynolds number is 4.46 million. The tests performed are pure sway, pure yaw, and static drift. Examples of the trajectory and orientation of the model are provided for each test in Fig. 7. The time-dependent tests have PMM periods of $T_{\mathrm{pmm}}=7.48$ seconds, a pure sway amplitude of $\eta_{0} / L=0.104$, and a pure yaw amplitude of $\psi_{0}=10.2$ degrees. These simulations replicate the experiments performed at the Iowa Institute of Hydraulic Research (IIHR) (Longo 

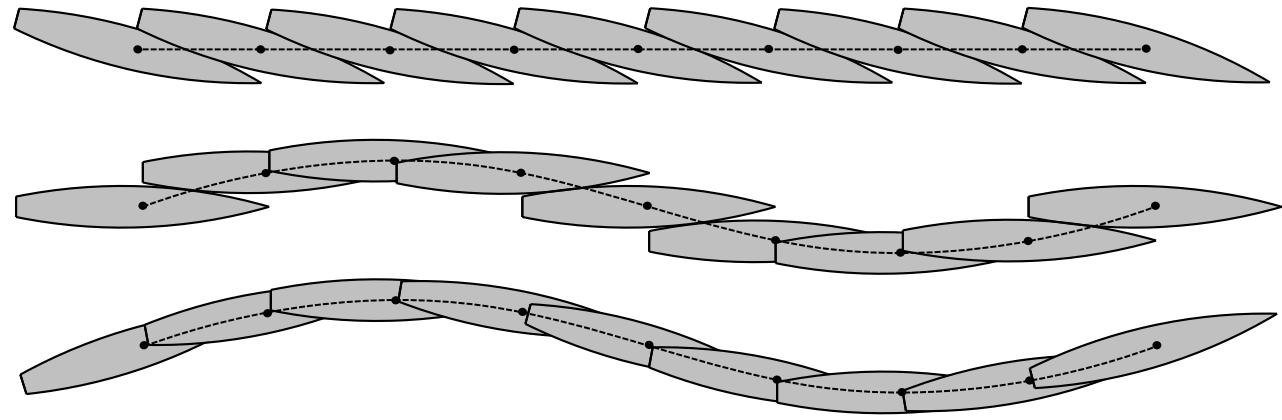

Figure 7: Top-to-bottom - static drift, pure sway, and pure yaw tests

et al., 2006). The model was fixed in sinkage and trim during the experiments. This section presents the results of the linearized URANS method compared to the experimental data as well as the computational expense of the simulations.

\subsection{Forces and moments}

The forces and moments presented in this section are the ship-fixed and non-dimensional axial surge force, lateral sway force, and yaw moment about midships $\left(X^{\prime}, Y^{\prime}\right.$, and $N^{\prime}$ from Eq. 24). Time histories are plotted over a single PMM period. Each plot contains two sets of data - experimental and linearized URANS simulations. Three grids are used to estimate the discretization error. The cell count and near-wall-layer metrics are summarized in Table 2.

Table 2: Grid characteristics

\begin{tabular}{lcc}
\hline & cells & $y_{\text {mean }}^{+}$ \\
\hline coarse & 202,000 & 60.1 \\
medium & 398,000 & 50.0 \\
fine & 918,000 & 41.7 \\
\hline
\end{tabular}

\subsection{Static drift with grid study}

The steady nature of the static drift test presents an opportunity to perform a grid study with the linearized URANS approach because it is straightforward to compute the time-averaged value of the steady force on the ship. 
The procedure presented in Eça and Hoekstra (2014) is employed in this work for a three-grid set of solutions. The first step is to identify the convergence condition using the solution on the coarse, medium, and fine grids. The solution on each grid is represented as $\phi_{3}, \phi_{2}$, and $\phi_{1}$, respectively:

$$
\begin{aligned}
& R=\frac{\phi_{2}-\phi_{1}}{\phi_{3}-\phi_{2}} \\
0<R<1 & \Rightarrow \text { Monotonic convergence } \\
-1<R<0 & \Rightarrow \text { Oscillatory convergence } \\
R>1 & \Rightarrow \text { Monotonic divergence } \\
R<-1 & \Rightarrow \text { Oscillatory divergence }
\end{aligned}
$$

Here, $R$ is the discriminating ratio of the solution on the three-grid set. In the simulations for the 5415 in static drift, the surge force displays monotonic divergence. This means that the error is not in the asymptotic range, and Richardson extrapolation is not used to estimate the error. It is believed that the divergence is related to the application of the wall-function on grids with varying near wall-layer thickness. It is not possible to systematically refine the grid and keep the first grid point off the wall in the same location, and this has important consequences for the wall shear stress prediction from the wall-function. The paper (Eça et al., 2015) has a clear presentation of the consequences of applying a wall-function to a turbulent flat plate with varying near wall-layer cell thickness.

However, the sway force and yaw moment show monotonic convergence. The observed order-of-accuracy is related to the discriminating ratio. If the solutions show convergence, and the observed order-of-accuracy is close to the theoretical, then Richardson's extrapolation may be used. For both the sway and yaw quantities, the observed order was much larger than the theoretical order. In this case, most steps in the algorithm are second-order, but there are first-order approximations in the gradient limiter and the gradient-boundary conditions.

To visualize the convergence of the solution, Fig. 8 shows the numerical data together with two curves. The curves correspond to a polynomial error model, where a least squares approach is used to solve for $\phi_{o}$ and $\alpha$ in Eq. 27. The blue-dashed curve represents the fit to Eq. 27 using the theoretical orderof-accuracy $p_{\text {th }}=2$, and the red-solid curve represents the fit using the observed order-of-accuracy $p$ that is calculated from the discriminating ratio (Eça and Hoekstra, 2014, Eq. 3): 


$$
\phi_{i}=\phi_{o}+\alpha\left(\frac{h_{i}}{h_{1}}\right)^{p}
$$

In Eq. 27, $\phi_{o}$ represents the extrapolated value (an estimated value corresponding to cell size $h_{i}=0$ ), and $\alpha$ is a constant coefficient. A representative cell size for each grid is denoted with $h_{i}$, where $h_{1}$ corresponds to the finest grid. The representative grid size is the cube root of the ratio of the volume of the flow domain and the number of cells.

The convergence behavior of each force and moment can be seen in Fig. 8 . Monotonic grid convergence can be seen for the sway force and yaw moment. Also, the extrapolated values of each quantity can be seen by inspection of the vertical axis as $h_{i} / h_{1} \rightarrow 0$.
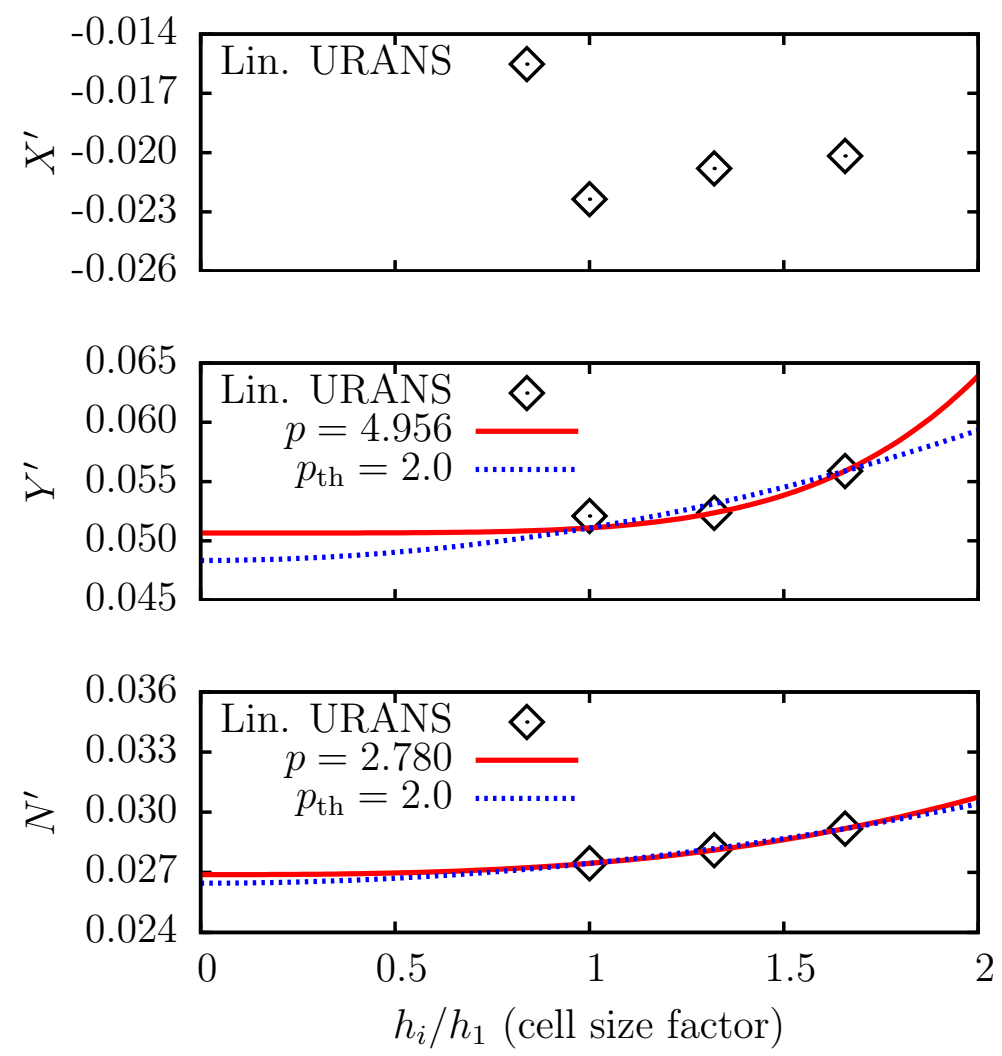

Figure 8: Grid study for static drift

Due to the monotonic divergence and the super-convergence displayed, an uncertainty assessment based on Richardson extrapolation is not reliable. 
In other words, the error is apparently not in the asymptotic range which is a fundamental assumption in the derivation of Richardson's extrapolation. Instead, a factor of safety is used as presented in Eq. 28.

$$
\begin{aligned}
U_{\phi} & =F_{s} \Delta_{M} \\
\Delta_{M} & =\max \left(\left|\phi_{\mathrm{i}}-\phi_{\mathrm{j}}\right|\right)
\end{aligned}
$$

The uncertainty is represented with $U_{\phi}$. The factor of safety is $F_{s}$, and $\Delta_{M}$ is the maximum difference in the solution among the grids. For the monotonic divergence of the surge force, it is suggested that $F_{s}=3$. The sway force and yaw moment show monotonic convergence and employ a lower factor of safety value, $F_{s}=1.25$. These findings are summarized in Fig. 9, where the fine grid results are plotted with the uncertainties.

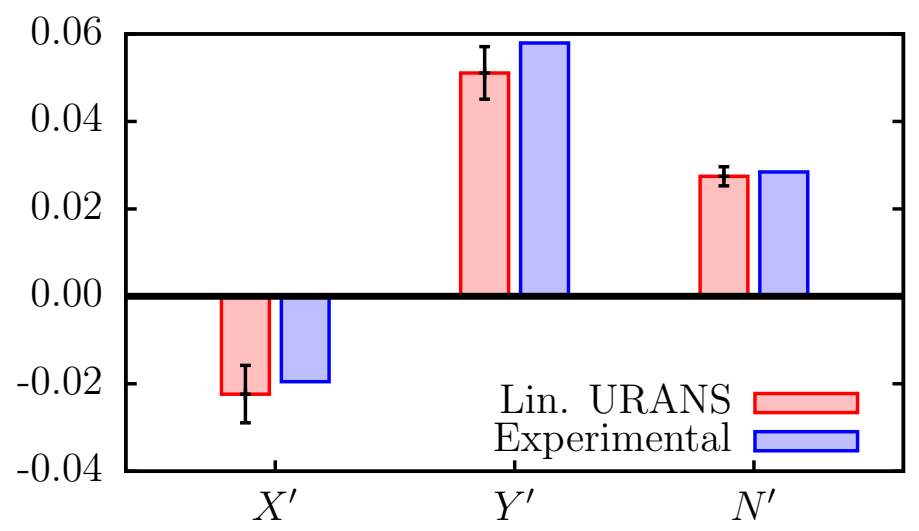

Figure 9: Force and moment uncertainties for static drift

Ship maneuvers often occur at low forward speed, and if the speed is low enough, the influence of the ship waves can be neglected. For example, if the length-based Froude number is less than approximately 0.1, the free-surface is often assumed to be flat and to act as a symmetry plane using the socalled zero-Froude-number approximation. To highlight the benefits of the linearized URANS method, the static drift simulation is repeated using the zero-Froude-number or double-body approximation. The coarse grid containing just over 200,000 cells is used with both approaches for the comparison.

Fig. 10 shows the major differences in the pressure solution at the $z=0$ calm-water plane. This can also be interpreted as the solution to the freesurface elevation since pressure and elevation are directly related to first-order 
as $p=\rho g \eta$. Both show a high pressure zone corresponding to the bow wave on the windward side, but the linearized URANS method exhibits gravity waves that are generated on the leeward side.
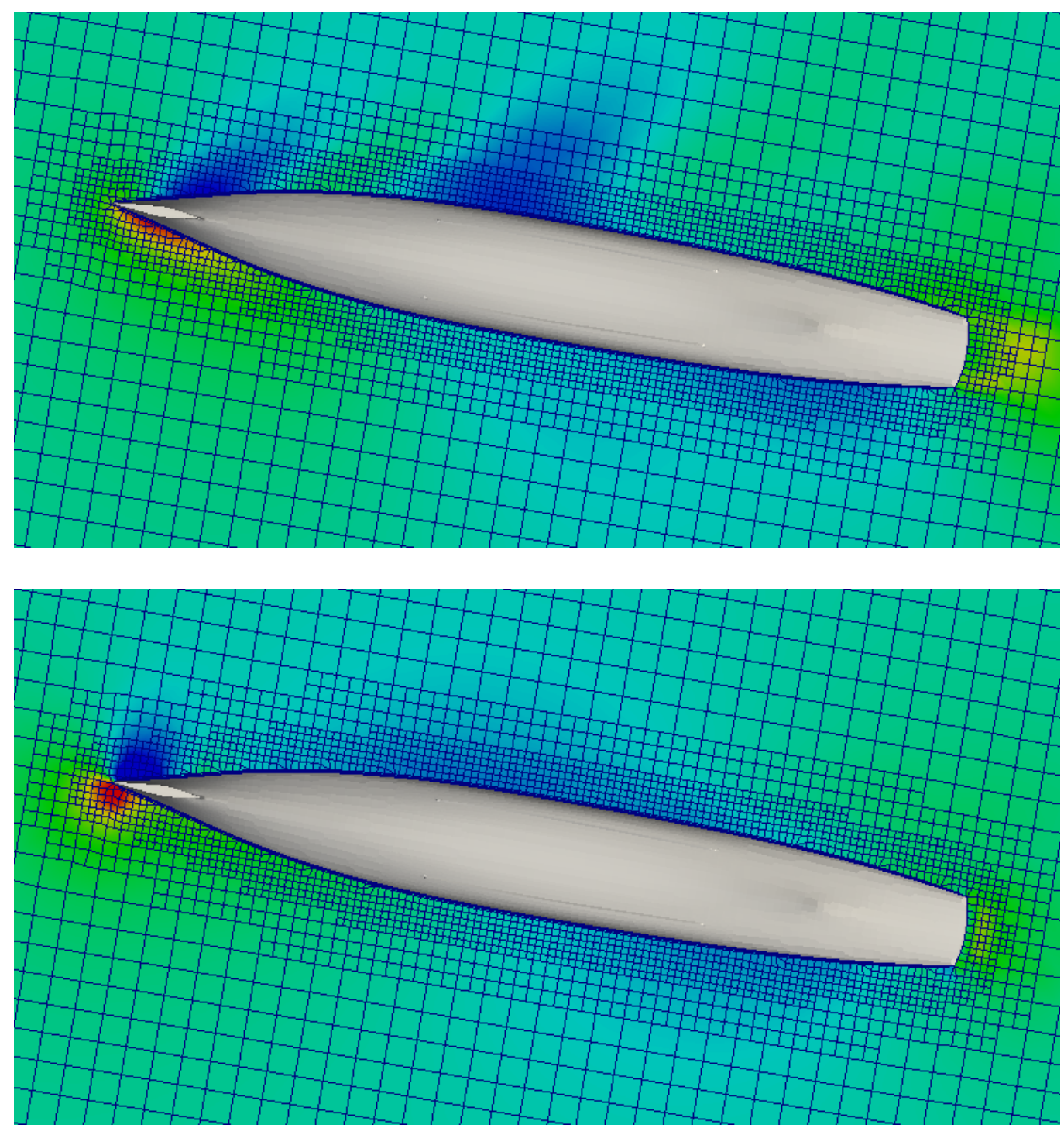

Figure 10: Coarse grid pressure comparison of linearized URANS (top) and double-body (bottom) for static drift $\beta=10^{\circ}$

For a more quantitative comparison, the forces obtained using both tech- 
niques are shown in Fig. 11. Both methods perform well in predicting the surge force obtained with the physical experiments. However, the zeroFroude-number approach shows a difference of over $20 \%$ for both the sway force and yaw moment. On the other hand, the linearized URANS method still compares well to the experiments being less than $5 \%$ different.

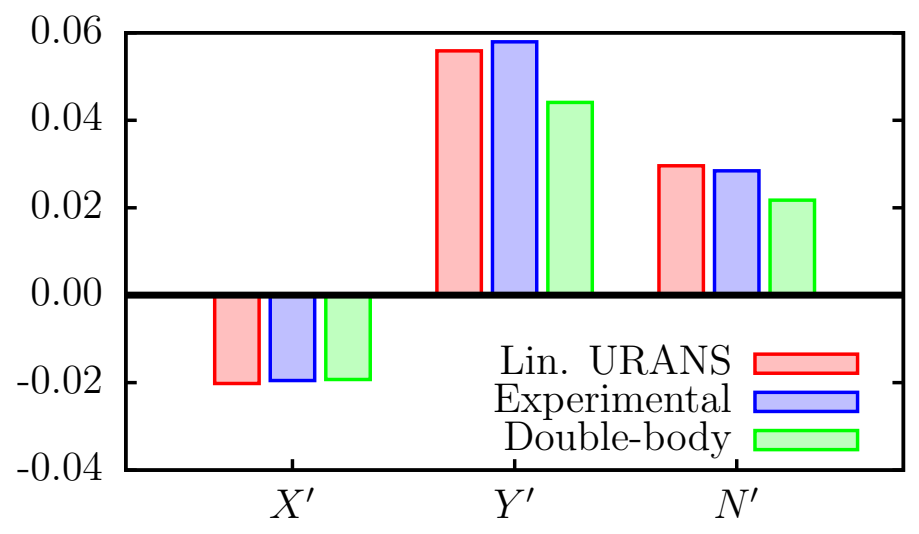

Figure 11: Static drift for linearized URANS and Double-body

The computational expense of each method is also important to compare. The linearized URANS method requires $10 \%$ more computational time than the double-body simulation. Specifically, 9.29 hours vs. 8.43 hours are needed on 12 processors for 60 seconds of simulated time. For a slightly greater expense, the improved accuracy of accounting for first-order wave effects is significant and still possible to obtain with computation on hardware that is available in a desktop workstation.

\subsection{Pure sway}

Fig. 12 presents the time series over one PMM period for the pure sway tests. The numerically predicted surge force shows a smaller amplitude than the experimental data. However, the time-averaged values are in good agreement. An important observation to make is the consistency between the three grids. It is uncommon for fully nonlinear methods to correspond so well with experiments using as few as 200,000 cells, as is being displayed here with the linearized URANS method (Bhushan et al., 2014). The sway force and the yaw moment are predicted well with the linearized approach. Other than 
a slight phase shift from the experiments, all grids provide accurate predictions. This phase shift may be present due to turbulence modeling, the wall-function, or the boundaries of the mesh. It may also be partly attributed to experimental error.
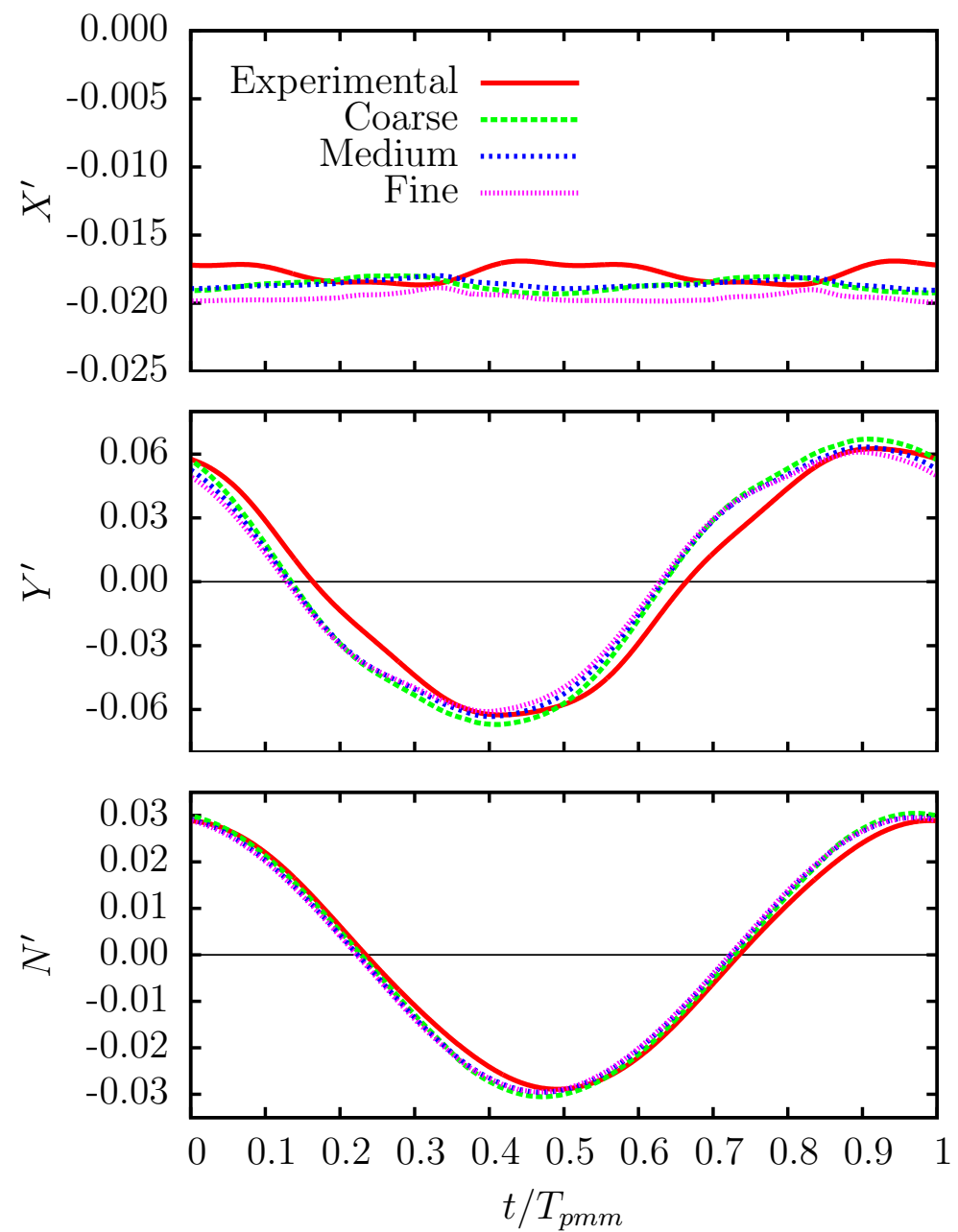

Figure 12: Forces and moments during pure sway

\subsection{Pure yaw}

Fig. 13 shows the forces and moment over one PMM period during the pure yaw test. Similar to pure sway, the surge force from the simulations differs from the experiments, but it should be noted that the solution exhibits 
convergence and the difference in the solution between the three grids is relatively small. The medium and fine grids are in very close agreement. The sway force and yaw moment agree well with the experiments, other than a small phase shift.
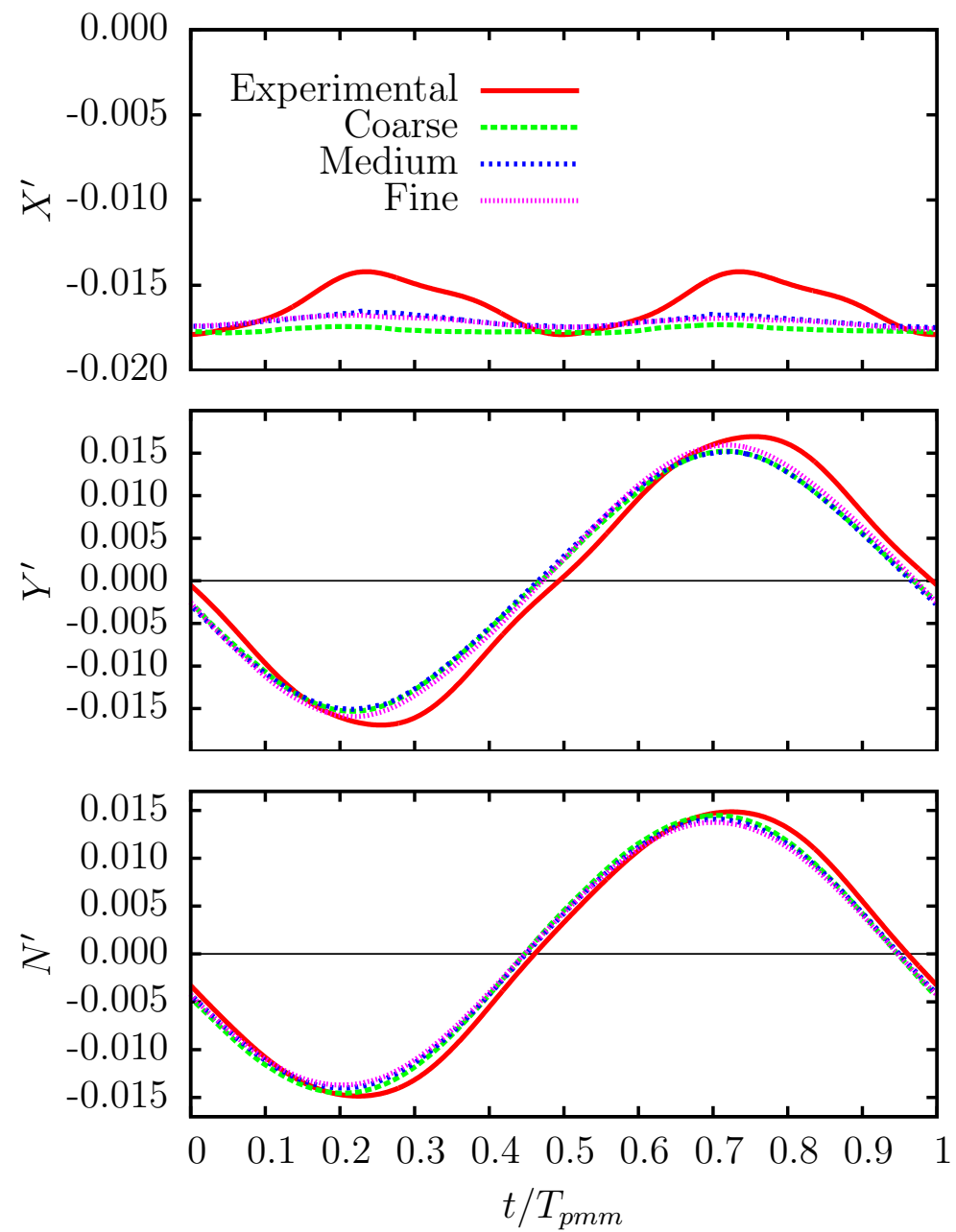

Figure 13: Forces and moments during pure yaw

\subsection{Computational time}

Computational efficiency is a primary goal of the linearized URANS methodology. The CPU expense is calculated by multiplying the walltime with the number of processors used to compute the solution over a single 
Table 3: CPU hours per PMM period

\begin{tabular}{lccc}
\hline & coarse & medium & fine \\
\hline pure sway & 11.5 & 56.3 & 221.9 \\
pure yaw & 10.4 & 35.8 & 196.0 \\
\hline
\end{tabular}

PMM period. This expense is reported in CPU hours for the dynamic maneuvers on each grid in Table 3 .

The low computational expense required by the linearized URANS method makes it suitable for use on a multi-core workstation. The ability to use machines already present in the workplace shows promise that RANS-based maneuvering simulations can be brought into engineering practice more immediately. It should also be noted that these CPU hours correspond to conservative simulations with no under-relaxation and maximum allowable Courant number of $C_{\max }=2$.

\section{Discussion and conclusions}

An unsteady linearized free-surface framework for a viscous, turbulent fluid is studied. Application of the technology focuses on ship maneuvering prediction, however, the general problem formulation is valid for a variety of ocean engineering problems. Two frames of reference are used to highlight the robustness of the method. The first is an inertial, body-fixed reference frame where governing equations are solved in either a steady or unsteady manner. The second variation is an inertial, Earth-fixed reference frame which solves the ALE form of the governing equations. Each variation produces similar results for a resistance test on a Wigley hull.

Within the overall linearized free-surface framework, a unique method for maneuvering prediction has been developed. This is an initial application and validation of the technology, but the results herein show the linearized URANS method to be a viable option when compared to model tests and fully nonlinear CFD. Although limited to the maneuvering assessment of a naval combatant in this article, application of the method can be extended to full ship forms with higher block coefficients and additional maneuvers such as turning circles and zig-zags. Utilizing a linear free-surface approximation and solving the URANS equations in the water phase shows promise in terms of both accuracy and efficiency. The reduced complexity in solving 
a first-order wave field compared to fully nonlinear approaches offers lower computational expense as seen with several PMM simulations on a DTMB 5415 model. Time-dependent maneuvers can be performed in less than 12 CPU hours. Even with coarse grids, forces and moments compare closely with experiments because the surface effects associated with maneuvering are accurately represented in a linearized framework.

\section{Acknowledgments}

The authors would like to gratefully acknowledge the U.S. Office of Naval Research program number N00014-13-1-0759 administered by Kelly Cooper. Additional acknowledgment goes to the Flux HPC Cluster provided by the University of Michigan Office of Research and operated by the High Performance Computing Group at the College of Engineering.

\section{References}

Bhushan, S., Xing, T., Visonneau, M., Wackers, J., Deng, G., Stern, F., Larsson, L., 2014. Post workshop computations and analysis for KVLCC2 and 5415. In: Larsson, L., Stern, F., Visonneau, M. (Eds.), Numerical Ship Hydrodynamics. Springer Netherlands, pp. 265-318.

Broglia, R., Dubbioso, G., Durante, D., Di Mascio, A., 2013. Simulation of turning circle by CFD: Analysis of different propeller models and their effect on manoeuvring prediction. Applied Ocean Research 39, 1-10.

Carrica, P. M., Sadat-Hosseini, H., Stern, F., 2012. CFD analysis of broaching for a model surface combatant with explicit simulation of moving rudder and rotating propellers. Computers \& Fluids 53, 117-132.

Cope, D. M., 2012. Design of a free-running 1/30th Froude scaled model destroyer for in-situ hydrodynamic flow visualization. Master's thesis, Massachusetts Institute of Technology.

Eça, L., Hoekstra, M., 2014. A procedure for the estimation of the numerical uncertainty of cfd calculations based on grid refinement studies. Journal of Computational Physics 262, 104-130. 
Eça, L., Saraiva, G., Vaz, G., Abreu, H., 2015. The pros and cons of wall functions. In: Proceedings of the ASME 2015 34th International Conference on Ocean, Offshore and Arctic Engineering. St. John's, Newfoundland and Labrador, Canada.

Gillespie, J. W., Daniels, A. S., Singer, D. J., 2013. Generating functional complex-based ship arrangements using network partitioning and community preferences. Ocean Engineering 72, 107 - 115.

Lindberg, O., Glimberg, S. L., Bingham, H. B., Engsig-Karup, A. P., Schjeldahl, P. J., 2013. Towards real time simulation of ship-ship interaction Part II: Double body flow linearization and GPU implementation. In: International Workshop on Water Waves and Floating Bodies (IWWWFB) 2013.

Longo, J., Yoon, H.-S., Toda, Y., Stern, F., 2006. Phase-averaged 3DPIV/wave elevations and force/moment measurements for surface compatant in PMM maneuvers. In: Proc. 26th ONR Symposium on Naval Hydrodynamics. Rome, Italy.

Newman, J. N., Tuck, E. O., 1974. Hydrodynamic interaction between two ships. In: Proceedings of the 10th Symposium on Naval Hydrodynamics. pp. 35-70.

Pinkster, J., Bhawsinka, K., 2013. A real-time simulation technique for shipship and ship-port interactions. In: International Workshop on Water Waves and Floating Bodies (IWWWFB) 2013.

Sclavounos, P. D., Thomas, B. S., 2007. Optimal control theory applied to ship maneuvering in restricted waters. Journal of Engineering Mathematics 58, 301-315.

Tanaka, I., 1983. Cooperative experiments on wigley parabolic models in Japan. Tech. rep., 17th ITTC Resistance Committee.

Toxopeus, S. L., 2009. Deriving mathematical manoeuvring models for bare ship hulls using viscous flow calculations. Journal of Marine Science and Technology 14, 30-38.

Ueno, M., Tsukada, Y., Kitagawa, Y., 2014. Rudder effectiveness correction for scale model ship testing. Ocean Engineering 92, 267 - 284. 
Wehausen, J., Laitone, E., 1960. Surface Waves. Vol. 9 of Encyclopedia of Physics: Fluid Dynamics III. Springer-Verlag, Berlin.

Woolliscroft, M. O., Maki, K. J., 2015. A linearized free-surface RANS method for unsteady ship maneuvering problems. In: International Workshop on Water Waves and Floating Bodies (IWWWFB) 2015.

Zhou, X., Sutulo, S., Guedes Soares, C., 2014. Computation of ship-to-ship interaction forces by a three-dimensional potential-flow panel method in finite water depth. Journal of Offshore Mechanics and Arctic Engineering 136. 